\title{
Remarkable Zeroth-Order Resonant Transmission of Microwaves through a Single Subwavelength Metal Slit
}

\author{
J. R. Suckling and J. R. Sambles \\ School of Physics, Exeter University, Devon, EX4 4QL, United Kingdom \\ C. R. Lawrence \\ QinetiQ, Cody Technology Park, Farnborough, GU14 OLX, United Kingdom
} (Received 15 February 2005; revised manuscript received 19 May 2005; published 28 October 2005)

A slit in a thick metal plate that is extremely subwavelength will not transmit microwaves polarized parallel to it. It is shown here that cuts perpendicular to the slit allow parallel polarized radiation to resonantly transmit. Furthermore, a zero-order mode may be excited within the slit, the frequency of which, to first order, is independent of the plate depth. Remarkably, for this novel type of resonance, the field in the slit oscillates with a constant phase and little amplitude variation throughout the plate depth, while the resonant wavelength tends to infinity as the slit width approaches zero.

Following the work of Ebbesen et al. [1], there is much interest in the transmission of electromagnetic radiation through subwavelength apertures in otherwise opaque screens. While the initial study [1] was for an array of holes in an opaque metal screen, much further work has been undertaken for the rather different geometry of narrow slits in metal screens [2-6]. The single slit case has also been studied [7-10] with incident radiation polarized perpendicular to the slit. Enhancement of transmitted signals through single slits or apertures has been demonstrated using surface modulation on both sides of the slit, or aperture, in both the optical [11] and microwave [12,13] regimes. It should be noted that these studies tend to focus just on grooves aligned parallel to the aperture that act to enhance the electric fields around the aperture and, hence, enhance transmission.

In this Letter, the resonant response of a single subwavelength slit modified by perpendicular cuts arranged in a periodic array along the length of the slit is studied for a range of frequencies and slit widths. Remarkably, it is shown that, even when the microwaves are polarized parallel to the slit, it is still possible to couple to Fabry-Perotlike resonant modes within the slit. Notably, this coupling is achieved at frequencies that would not normally be allowed to propagate through the slit at all. The frequencies of these modes are no longer simply determined by the plate depth as expected from Fabry-Perot theory. Indeed, it is possible to couple to a zero-order Fabry-Perot mode which has a resonant frequency almost completely independent of plate depth. This is very surprising, as it is impossible to couple to this mode when the microwaves are polarized perpendicular to the slit. Furthermore, this zero-order resonant mode can exist at wavelengths that are many times larger than any resonant dimensions within the structure, indicating the possibility of fabrication of arrays with strong transmission of very long wavelengths through subwavelength elements. It should be noted that this is distinct from transmission through an unadorned subwavelength slit [7,9], where the resonant dimension is the depth of the slit and the longest resonant wavelength is simply twice the depth of the slit; here the zeroth-order mode can resonate at wavelengths many times greater than any of its resonant dimensions. It is shown that it is the manner in which the microwaves couple into the slit that allows the zero-order mode to exist and that the coupling mechanism is fundamentally different to the process exhibited by a simple slit [11-13].

Fabry-Perot theory predicts that the resonant frequency $f_{N}$ of a cavity formed by a slit in a metal plate of depth $t$ is given by Eq. (1), where $N$ is the mode number and $c$ the speed of light:

$$
f_{N}=\frac{c N}{2 t} .
$$

In the case of a single slit in a metal screen, these modes will be supported only if the electric field is polarized perpendicular to the slit (a subwavelength slit, with width $<\lambda / 2$, illuminated with parallel polarized photons can support only evanescent electromagnetic fields decaying away from the illuminated surface and will not resonantly transmit). An infinitely long single slit has been shown to resonate at a frequency approximated by Eq. (1), and, when the slit is made finite in length, the resonant frequency is shown to rise [9].

The experimental slit sample with periodic perpendicular cuts is shown in Fig. 1. The slit is formed by the gap between two large aluminum plates, each with a face area $400 \times 200 \mathrm{~mm}$. These plates are separated by smooth dielectric spacers which are placed outside the beam spot. These spacers allow a wide range of slit widths $w_{s}$ to be characterized $(30-1000 \mu \mathrm{m})$. Perpendicular cuts, with a depth $d$ of $3 \mathrm{~mm}$ and a width $w_{c}$ of $0.8 \mathrm{~mm}$, are periodically spaced along the slit, with a pitch $p$ of $7.1 \mathrm{~mm}$. The slit is placed directly between two microwave horns, 


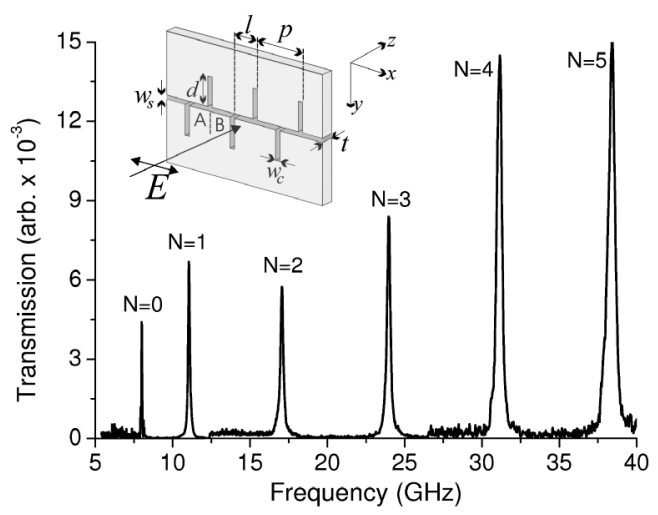

FIG. 1. Transmission spectrum of the sample (inset) with resonant mode numbers indicated. Inset: Illustration of a section of the sample, labeled with the dimensions and incident microwave direction and polarization.

one acting as a source and the other as a detector. The detector horn is placed $\sim 10 \mathrm{~cm}$ distance from the plate, far enough away not to be considered near-field yet as close as possible to give acceptable signals from the very narrow slits. The horns are aligned such that the incident microwaves are polarized parallel to the slit and detected in the same polarization. Then the transmission spectrum is measured from 5.2 to $50 \mathrm{GHz}$ (wavelength $6<\lambda<58 \mathrm{~mm}$ ) for a range of plate depths. Over nearly all of this range of wavelengths, both the slit and cut dimensions are below the wavelength conditions for waveguiding in the cuts, which begins to occur above $\sim 49.9 \mathrm{GHz}$. An example resonant transmission spectrum is given in Fig. 1; it shows the resonant peaks that are plotted in subsequent figures as points. Note that the spectrum is comprised of five frequency ranges, with data acquired with five different horn sources and detectors. It is interesting to note that the absolute transmission is of the same order as that observed in Refs. [7,9], in which the transmission of microwaves polarized perpendicular to a slit is studied.

Figure 2 shows the resonant frequency response of the structure as a function of slit depth (metal plate depth) $t$ at a fixed slit width $\left(w_{s}=50 \mu \mathrm{m}\right)$. (For this experiment, the cuts on opposite sides of the slit are in antiphase; i.e., they are offset by half the cut spacing $p$, as shown in Fig. 1.) To explore the depth dependence of the modes, five different samples with plate depths $19.63,9.95,2.95,1.20$, and $0.71 \mathrm{~mm}$ are studied. The output from a finite element method (FEM) computer model, Ansoft's high frequency structure simulator (HFSS) [14], is also plotted for a number of plate depths. The predicted FEM model frequencies for 19.63 and $9.95 \mathrm{~mm}$ plate depths show very close agreement with the experimental data. The most remarkable feature of Fig. 2 is the lowest resonant mode $(N=0)$ $(\sim 8 \mathrm{GHz}$ at $t=19.63 \mathrm{~mm})$ whose frequency is almost completely independent of plate depth. This depth independence is consistent with the existence of a mode with almost constant electric field strength and phase through-

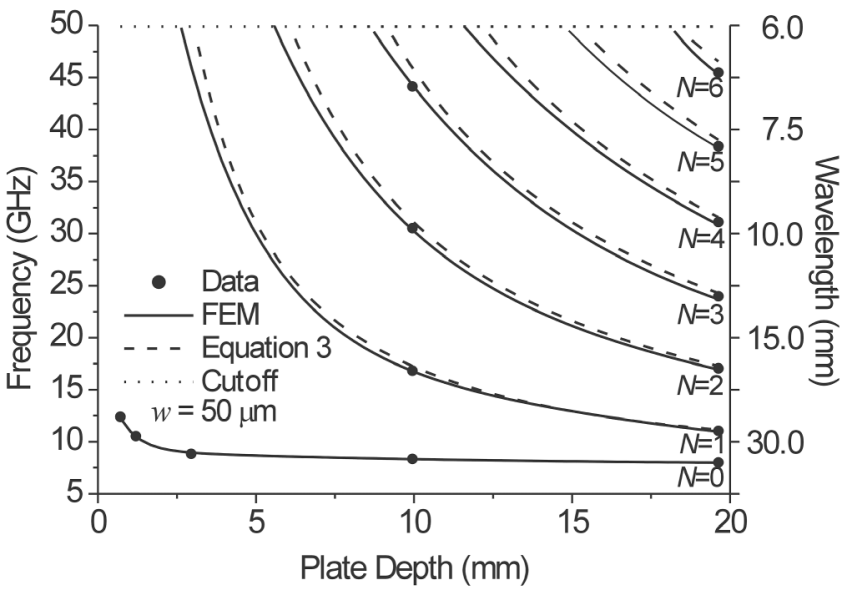

FIG. 2. Experimental data of resonant frequency against plate depth (0.71-19.63 mm) for a slit width $w_{s}$ of $50 \mu \mathrm{m}$ (dots). FEM modeling (solid line) for $t=0.71-19.63 \mathrm{~mm}$ and predictions from Eq. (2), using $f_{0}$ values as predicted by the FEM model (dashed line). Frequency at which the cuts waveguide is $49.9 \mathrm{GHz}$ (dotted line).

out the entire depth of the slit. This field configuration may be considered as the zero-order Fabry-Perot mode. The rise in frequency of this mode at very low slit depths is due to end effects. The higher order modes $(N=1,2,3$, etc.) ( $\geq 11 \mathrm{GHz}$ at $t=19.63 \mathrm{~mm})$, which are more FabryPerot-like in nature, show the expected rise in frequency as $t$ is reduced. No data is presented above $49.9 \mathrm{GHz}(\lambda \sim$ $6 \mathrm{~mm}$ ), as beyond this frequency the cuts act as individual, isolated resonant waveguides and the transmission due to the slit is lost to the more intense waveguide transmission. The following equation models the resonant frequencies of the sample:

$$
f_{N}=\frac{c}{2 \pi}\left[\left(\frac{N \pi}{t}\right)^{2}+f_{0}{ }^{2}\right]^{1 / 2} .
$$

Here $f_{0}$ is the resonant frequency of the zero-order mode and represents a perturbation of the normal Fabry-Perot frequencies. While it is beyond the scope of this Letter to quantitively explore in detail the dependence of $f_{0}$ on the sample dimensions, it is possible to outline an analytic approximation of the structure, the equivalent circuit (EC) approximation. An EC represents the slit and cuts as a circuit comprised of inductors and capacitors (LC). The incident microwaves create an electric current on the surface of the metal parallel to the polarization direction. The cuts perturb the flow, creating partial current loops around them, thereby inducing strong magnetic fields within the cut [Fig. 3(a)]; the cuts are thus predominantly inductive. The magnetic field for the zero-order mode is "up" one cut and "down" the next, on the opposite side of the slit. There is also a charge distribution that creates strong electric fields within the slit which approximately acts as a capacitor. As electric fields are constrained to be perpendicular to a metal surface, the electric vector has to 


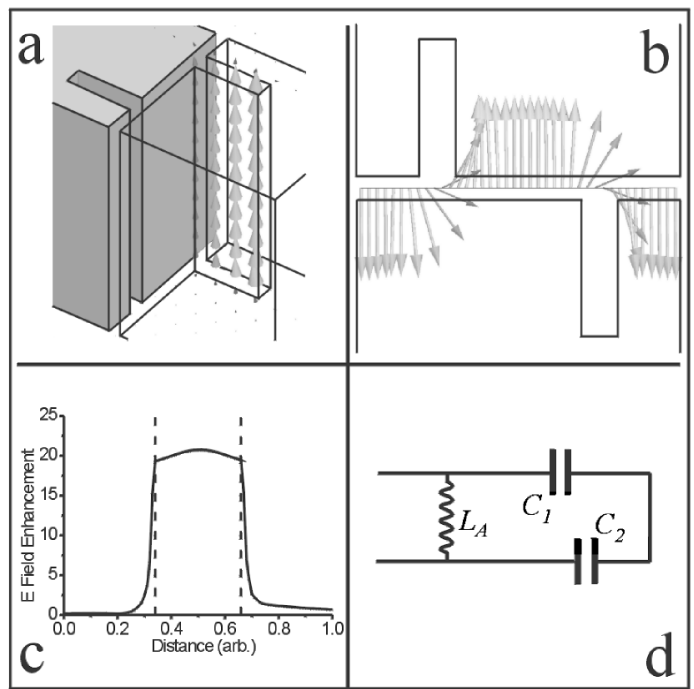

FIG. 3. FEM model data. (a) Instantaneous magnetic field direction within a cut, (b) instantaneous electric field direction along the slit, and (c) time averaged electric field magnitude along the depth of the slit on resonance of the zero-order mode $(17.29 \mathrm{GHz})$ for a slit width of $500 \mu \mathrm{m}$ and plate depth of $9.95 \mathrm{~mm}$. (d) EC used as the basis Eq. (3).

undergo a $90^{\circ}$ rotation in orientation on entering the slit from a cut to satisfy the boundary conditions of the slit and the charge distribution on the walls of the slit. These electric fields are thus opposite in direction on either side of a cut, while being equal in magnitude [Fig. 3(b)], with a slight sinusoidal variation in magnitude along the length of slit. The fields plotted are for the zeroth-order resonance within the $9.95 \mathrm{~mm}$ deep slit, $5 \mathrm{~mm}$ into the slit, at a slit width of $500 \mu \mathrm{m}$ and a frequency of $17.29 \mathrm{GHz}$. Figure 3(c) shows the time averaged electric field enhancement throughout the depth of the slit $(9.95 \mathrm{~mm})$ and for $10 \mathrm{~mm}$ either side in the surrounding air. Note that the field is relatively constant along the entire depth; crucially, the resonance does not have a node.

Figure 3(d) shows an equivalent circuit layout which may represent the slit and cuts. The cuts are predominantly inductive $\left(L_{A}\right.$ in Fig. 3), and the slit approximates to separate parallel plate capacitors between each cut $\left(C_{1}\right.$ and $C_{2}$ in Fig. 3). This then leads to an approximate form of the resonant frequency being expressed as

$$
f_{0}=\left(\frac{w_{s} p}{\pi^{2} \varepsilon_{0} L_{A}\left(p^{2}-l^{2}\right)}\right)^{1 / 2},
$$

where $L_{A}$ is the inductance of the cuts and the slit capacitances are expressed in terms of the sample dimensions $p$ and $w_{s} . l$ is the offset of the cuts in one plate relative to those in the other (Fig. 1).

Figure 4 shows the change of resonant frequency of modes $N=0,1,2,3$ within the $9.95 \mathrm{~mm}$ deep plate with slit width $\left(30 \leq w_{s} \leq 1000 \mu \mathrm{m}\right)$. Also shown is the cutoff frequency of the cuts $(\sim 49.9 \mathrm{GHz}$, corresponding to $\lambda=$

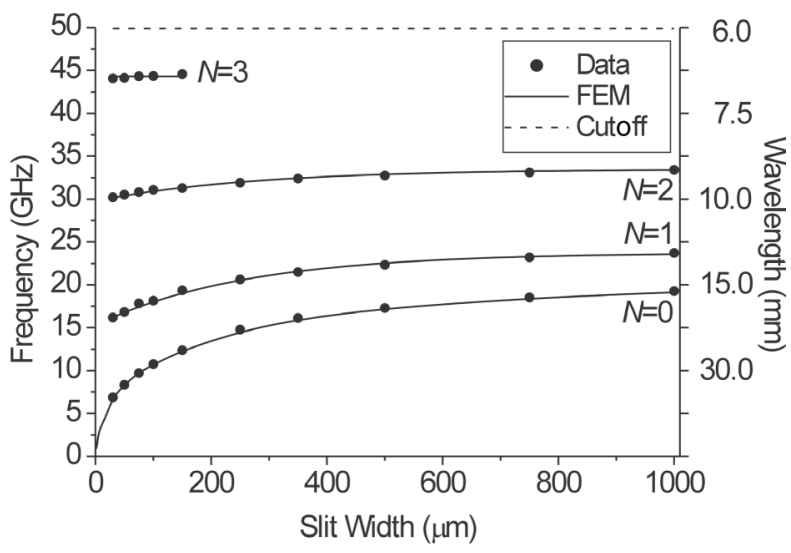

FIG. 4. Experimental data of resonant frequency against slit width $(30-1000 \mu \mathrm{m})$ for a plate depth $t$ of $9.95 \mathrm{~mm}$ (circles). FEM modeling (solid line). Frequency at which the cuts waveguide is $49.9 \mathrm{GHz}$ (dashed line).

$2 d$ ), above which they transmit as waveguides for polarization along the slit. (The $N=3$ mode is not plotted for all slit widths as its wide resonant peak becomes perturbed by the cutoff edge for a slit width greater than $\sim 150 \mu \mathrm{m}$.) The resonant frequencies all decrease on reduction of the slit width, with the zero-order mode tending to zero frequency. These decreases in frequency correspond to a flattening of the sinusoidal electric field profile within the slit between the cuts and a simultaneously increasing phase change of the electric field under each cut [Fig. 3(b)]. At the limit of zero frequency, the field profile along the slit may be considered spatially invariant between the cuts with a $\pi$ radians phase change underneath each cut. Unfortunately, through absorption by the metal (as observed in Ref. [7] and due to the finite conductance of the metal at microwave frequencies), the transmitted mode broadens and the signal from a single slit becomes too weak to measure accurately at frequencies below about $6.7 \mathrm{GHz}$; note that the computer modeling does not suffer from noise on the signal and continues to predict the resonant frequency almost to $0 \mathrm{GHz}$. The lowest experimental frequency corresponds to a wavelength of $43.4 \mathrm{~mm}$, 4 times the length of any resonant dimension. If it were not for absorption by the metal, the structure would strongly transmit wavelengths far longer than this. It is also interesting to note that the drop in resonant frequency with slit width is in contradiction to the effect predicted in Ref. [7], indicating that changes in resonant frequencies due to open end effects of the slit are minor compared to the phase compression under the cuts.

The above results concerned a slit with the plates set such that the cuts on opposing plates were separated by $3.55 \mathrm{~mm}$ along the slit $\left(180^{\circ}\right.$ out of phase). The influence of changing this phase (altering the lateral offset $l$ ) has also been studied. It might be expected that each resonant mode would split into two modes, one descending in frequency, the other rising, corresponding to the two different length 


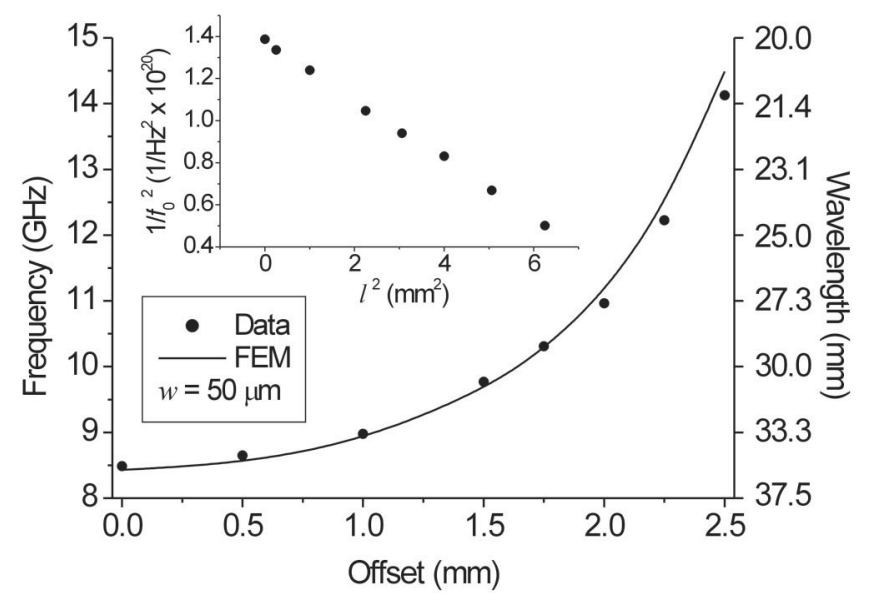

FIG. 5. Experimental data of resonant frequency shift against lateral offset $l$ of $0-2.5 \mathrm{~mm}$ for a slit width $w_{s}$ of $50 \mu \mathrm{m}$ (circles). FEM modeling (solid line). Inset: Replot of experimental data as predicted by Eq. (3).

resonant cavities formed between adjacent pairs of cuts ( $A$ and $B$ in Fig. 1). Figure 5 shows that this is not the case with the resonant mode rising in frequency with increase in lateral offset $(0 \leq l \leq 2.5 \mathrm{~mm})$ for the $9.95 \mathrm{~mm}$ deep plate. The inset in Fig. 5 shows the prediction of the LC $\mathrm{EC}$ [Eq. (3)]. Altering the offset of the cuts is equivalent to increasing slit capacitance in between two cuts, while simultaneously decreasing it between the next two cuts. Plotting $1 / f_{0}^{2}$ against $l^{2}$ should yield a straight line. This inset result in Fig. 5 shows that this approximate, LC EC approach is valid.

In summary, resonant transmission of microwaves polarized parallel to a single slit in metal with perpendicular cuts has been recorded for wavelengths substantially exceeding all resonant structure dimensions. (Although the sample investigated in this work was resonant in the microwave regime, further FEM modeling suggests that the phenomenon can be recreated at optical frequencies, although the enhanced absorption of the metal in this frequency regime will severely limit the transmission at very narrow slit widths.) It has been shown that the cuts provide an inductive coupling mechanism that allows microwaves to enter the slit. This coupling also enables access to the zero-order Fabry-Perot mode, which is im- possible with a simple unadorned slit for either microwave polarization. The frequency of the resonant modes has been shown to be controlled by the dimensions of the structure in such a way that the resonant wavelength tends to infinity as the width of the slit tends to zero. Remarkably, very thin plates (depth « wavelength) show this same effect. An equivalent circuit analogy has been shown to describe the resonant response of the structure very well as a first approximation. Further, the slit and cuts have been shown to resonate like pairs of coupled oscillators if one plate is moved laterally with respect to the other.

The authors thank Mr. P. Cann for his assistance in the construction of the samples and both the Engineering and Physical Sciences Research Council (EPSRC) and QinetiQ for their financial support.

[1] T. W. Ebbesen, H. J. Lezec, H.F. Ghaemi, T. Thio, and P. A. Wolff, Nature (London) 391, 667 (1998).

[2] J. A. Porto, F. J. Garcia-Vidal, and J. B. Pendry, Phys. Rev. Lett. 83, 2845 (1999).

[3] S. Astilean, Ph. Lalanne, and M. Palamaru, Opt. Commun. 175, 265 (2000).

[4] S. Collin, F. Pardo, T. Teissier, and J.-L. Pelouard, J. Opt. A Pure Appl. Opt. 4, S154 (2002).

[5] H. E. Went, A. P. Hibbins, J. R. Sambles, C. R. Lawrence, and A. P. Crick, Appl. Phys. Lett. 77, 2789 (2000).

[6] A.P. Hibbins, J. R. Sambles, C. R. Lawrence, and D. M. Robinson, Appl. Phys. Lett. 79, 2844 (2001).

[7] Y. Takakura, Phys. Rev. Lett. 86, 5601 (2001).

[8] F. Yang and J. R. Sambles, Phys. Rev. Lett. 89, 063901 (2002).

[9] J. R. Suckling, J. R.Sambles, A. P. Hibbins, M. J. Lockyear, and C. R. Lawrence, Phys. Rev. Lett. 92, 147401 (2004).

[10] J. Bravo-Abad, L. Martin-Moreno, and F. J. Garcia-Vidal, Phys. Rev. E 69, 026601 (2004).

[11] H. J. Lezec, A. Degiron, E. Devaux, R. A. Linke, L. Martin-Moreno, F. J. Garcia-Vidal, and T. W. Ebbesen, Science 297, 820 (2002).

[12] A.P. Hibbins, J. R.Sambles, and C. R. Lawrence, Appl. Phys. Lett. 81, 4661 (2002).

[13] M. J. Lockyear, A. P. Hibbins, J. R. Sambles, and C. R. Lawrence, Appl. Phys. Lett. 84, 2040 (2004).

[14] HFSS, Ansoft Corporation, Pittsburgh, PA, USA. 